\title{
Anogenital warts and other HPV-associated anogenital lesions in the HIV-positive patient: a systematic review and meta-analysis of the efficacy and safety of interventions assessed in controlled clinical trials
}

\author{
Ricardo Niklas Werner, Lukas Westfechtel, Corinna Dressler, Alexander Nast
}

- Additional material is published online only. To view please visit the journal online (http://dx.doi.org/10.1136/ sextrans-2016-053035).

Division of Evidence-based Medicine (dEBM), Department of Dermatology, Venereology and Allergology, CharitéUniversitätsmedizin Berlin, Berlin, Germany

\section{Correspondence to} Dr Ricardo Niklas Werner, Klinik für Dermatologie, Venerologie und Allergologie, Division of Evidence based Medicine (dEBM), CharitéUniversitätsmedizin Berlin, Charitéplatz 1, 10117 Berlin, Germany;

ricardo.werner@charite.de

Received 5 December 2016 Revised 12 April 2017 Accepted 22 April 2017 Published Online First 20 June 2017
CrossMark

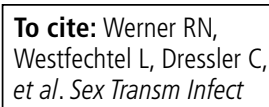

\section{ABSTRACT}

Objectives Anogenital warts (AGW, condylomata acuminata) and intraepithelial neoplasia (IEN) do not only impact health and social well-being, they are also associated with considerable costs for the healthcare systems. Immunocompromised and HIV-positive patients carry the highest epidemiological burden of human papillomavirus (HPV) infection and comprise a population specifically susceptible to treatment failures and recurrences. This systematic review aimed at identifying and appraising the available evidence from controlled studies of interventions for the treatment of AGW and IEN in immunocompromised patients.

Methods We conducted a comprehensive literature search. The Cochrane Collaboration's tool was used to assess risk of bias in included studies. Our confidence in the (pooled) effect-estimates was evaluated according to the Grading of Recommendations Assessment, Development and Evaluation approach. All evaluations were based on data independently extracted by two review authors. Results Nine randomised controlled trials and two controlled studies were eligible, investigating external AGW, intra-anal and/or vaginal warts, and intra-anal and/or perianal IEN. The identified studies assessed imiquimod, cidofovir, fluorouracil, electrocautery, systemic interferon- $\alpha$ and interferon- $\beta$, and the combination of intralesional interferon- $\alpha$ and podophyllin. Four studies combined an ablational intervention with either imiquimod, cidofovir, intralesional or systemic interferon$\alpha$. One study investigated an experimental therapeutic vaccination (HPV 16 E7) at different concentrations. Conclusions The quality of the evidence ranged from 'very low' to 'moderate' and was limited by the often small samples. Evidence was available for the efficacy of electrocautery for intra-anal IEN, and imiquimod cream for external AGW. Some further interventions should be subjected to investigations in larger samples. No data on some interventions established for the treatment of AGW in immunocompetent patients such as podophyllotoxin, sinecatechins, laser ablation or trichloroacetate were available. Future trials should address these gaps and include relevant patient-reported outcomes such as health-related quality of life.

\section{INTRODUCTION}

Anogenital human papilloma virus (HPV) infection is among the most common STIs, with an incidence of 38.4 genital HPV infections per 1000 personmonths in HIV-negative men. ${ }^{\text {1 }}$ Based on the HPV-DNA positivity of smear samples, the prevalence of infection in 18-40-year-old men who reported having had heterosexual intercourse only was found to be $71.2 \%$ at any anogenital site, $16.6 \%$ in the anal canal and $21.3 \%$ in the perianal region in one study. ${ }^{\text {w2 }} \mathrm{HPV}$ infection rates are higher in men who have sex with men (MSM) ${ }^{\text {w3 }}$ and particularly HIV-positive MSM. ${ }^{\text {w4 }}$ Likewise, the manifested forms of anogenital HPV infection, that is, anogenital warts (AGW, condylomata acuminata) and intraepithelial neoplasia (IEN), are common ${ }^{\text {w5-9 }}$ and impose considerable costs to the healthcare system. $^{\text {w10-15 }}$

AGW may cause discomfort and severe distress in affected patients, resulting in significant loss in quality of life (QoL). ${ }^{\mathrm{w} 16-21}$ IEN, moreover, potentially progress concerning grade of dysplasia and may develop to invasive squamous cell carcinoma. ${ }^{\text {w22 }}{ }^{23}$ Progression rates are exceptionally high in HIV-positive and immunosuppressed patients. ${ }^{\text {w24-28 }}$

Generally, low-risk HPV types are acknowledged as causative agents for AGW, which can often be distinguished from IEN on the basis of their clinical appearance. However, there is a substantial rate of mislabelling, particularly in HIV-positive patients. ${ }^{\text {w29 }}$ ${ }^{30}$ Recent data question whether the strict differentiation of low-risk and high-risk HPV types may be maintained. ${ }^{\text {w31 }}$

Since anogenital HPV infections are contagious, associated with low spontaneous clearance rates, significantly impact QoL and potentially progress to invasive disease, it is relevant to identify effective treatment strategies. The goal of this systematic review and meta-analysis was to systematically identify and appraise evidence from controlled clinical studies on the efficacy and safety of interventions investigated for the treatment of anogenital HPV-induced lesions in immunocompromised and HIV-seropositive patients.

\section{METHODS}

This review was part of a three-phase project (Part one: self-administered interventions for anogenital warts in immunocompetent patients: a systematic review and meta-analysis. doi: 10.1136/sextrans2016-052768). ${ }^{\mathrm{w} 32}$ In part 3 , the use of systemic 
interferon (IFN) as an adjuvant treatment for anogenital warts was investigated. Similarities throughout the methods section may occur. We followed the methods recommended by Cochrane ${ }^{\text {w33 }}$ and the 'Grading of Recommendations Assessment, Development and Evaluation' (GRADE) approach. ${ }^{\text {w34 }}$ The review protocol has been published (http://www.crd.york.ac.uk/PROSPERO/display_ record.asp? $\mathrm{ID}=\mathrm{CRD} 42016041212$ ).

\section{Eligibility criteria}

Study design was restricted to randomised controlled trials (RCTs) and clinically controlled, non-randomised parallel-group studies (CCTs). Abstracts were included if sufficient information was reported therein; language was restricted to English, German and French.

Studies had to report on immunocompromised and/or HIV-seropositive patients with a clinically or histologically confirmed diagnosis of HPV-associated benign or premalignant anogenital disease at baseline. This included AGW and anogenital IEN, comprising specific forms such as bowenoid papulosis, erythroplasia of Queyrat or Bowen's disease. Study populations were restricted to HIV-seropositive and/or immunocompromised patients with AIDS, iatrogenic immunosuppression (eg, transplant recipients) or other concomitant diagnoses associated with a reduced immunological function (eg, haematological disease). If studies separately reported on a subgroup according to these criteria, these data were also included. Studies investigating exclusively cervical or urethral localisation of AGW/IEN or invasive disease were not included.

Any intervention or combination of interventions directed at the elimination or reduction of the HPV-associated anogenital disease burden was included. This included the unlabelled use of commercial products and/or the investigational use of products not yet approved. Any of the included interventions, placebo preparations, vehicle or no treatment could serve as comparator.

At least one of the following outcomes had to be reported:

- complete clearance (CC) at 4 weeks ( \pm 4 weeks) ['shortterm-CC'],

- or at 24 weeks ( \pm 16 weeks) after the end of treatment (EOT) ['intermediate-term-CC'],

- dropouts due to adverse events (AEs).

The following outcomes were considered if available:

- pain;

- local AE;

- QoL;

- recurrence of lesions at 24 weeks ( \pm 16 weeks) ['intermediate-term-recurrence'] or at 12 months ( \pm 2 months) after EOT ['long-term-recurrence'] in responders who had a $\mathrm{CC}$ at EOT;

- CC at 12 months after EOT ( \pm 2 months) ['long-term-CC’]

- reduction of disease severity (reduction in lesion counts, lesion area or grade of dysplasia).

\section{Data management}

We searched the Cochrane Library, Embase and MEDLINE (see online supplementary material for search strategy). Reference lists of included studies were screened.

Two investigators (RNW, LW) independently screened titles and abstracts for eligibility, evaluated included titles/abstracts in full text and extracted data (see online supplementary material for data items). Disagreements concerning inclusion of studies or extracted data were solved by discussion or by involving a third investigator (CD/AN). For efficacy outcomes, data according to the intention-to-treat population were preferred; if these were not reported, a non-responder imputation was applied for CC rates.

Review Manager ${ }^{\mathrm{w} 35}$ was used to calculate risk ratios (RRs), ${ }^{\mathrm{w} 36}$ including 95\% CIs. When pooling was appropriate (ie, absence of clinical heterogeneity regarding interventions/patients and presence of sufficient statistical homogeneity $\left.\left(\mathrm{I}^{2} \leq 60 \%\right)\right)$, we performed Mantel-Haenszel meta-analyses, applying a random-effects model (DerSimonian and Laird). ${ }^{\text {w3 }}$

\section{Risk of bias and overall quality of evidence}

The GRADE approach ${ }^{\text {w37 }}$ was used to evaluate the overall quality of evidence for each available outcome: the risk of bias of each included study was assessed with the 'Cochrane Collaboration's tool'. ${ }^{38}$ The overall confidence in the effect estimates ${ }^{\mathrm{w} 39}$ was evaluated on the outcome level and categorised as 'very low', 'low', 'moderate' or 'high', under consideration of the risk of bias of contributing studies, ${ }^{\mathrm{w}} 40$ inconsistency, ${ }^{\mathrm{w}} 41$ indirectness, ${ }^{\mathrm{w} 42}$ imprecision ${ }^{\mathrm{w} 43}$ and publication bias. ${ }^{\mathrm{w} 44}$ Criteria for the evaluations are detailed in the online supplementary material.

\section{RESULTS}

The search (10 June 2016) produced 1096 hits. Of 893 unique hits, 846 were excluded during the title/abstract screening, leaving 47 records for full-text assessment. Ten study reports were eligible for data extraction. One additional publication was identified in the references of included studies (for study selection process, see figure 1; see online supplementary material for detailed reasons for excluding studies during the full-text assessment).

The 11 eligible studies ${ }^{1-11}$ included a total of 506 participants (5-65 per study group). All studies comprised participants who were immunocompromised due to HIV-positivity. Four studies ${ }^{2}$ 9-11 were not specifically designed to include HIV-positive participants, but separately reported on this subgroup. Online supplementary table S1 gives an overview of the study characteristics. One study was not included in the quantitative analysis due to the low number of participants in the study groups. ${ }^{7}$

The risk of bias was heterogeneous (figure 2) and is further described in the online supplementary material. Seven studies ${ }^{1} 256^{9-11}$ were rated as being at high risk of 'other sources of bias', which are discussed below.

More detailed results for the comparisons listed below are given in the online supplementary material, including GRADE - 'Summary of findings' - tables with reasons for the quality of evidence evaluations.

\section{Imiquimod versus placebo}

Two RCTs compared imiquimod 5\% cream with placebo; one study ${ }^{4}$ in patients with external AGW of different locations and one study ${ }^{3}$ in patients with high-grade anal intraepithelial neoplasia (AIN). Both trials did not demonstrate statistically significant effects on short-term CC (pooled RR 2.34, 95\% CI 0.68 to $8.04, \mathrm{I}^{2}=0 \%$, GRADE: low). A reduction in disease severity was achieved more often with imiquimod regarding partial clearance $(\geq 50 \%$ clearance of baseline AGW, RR 2.69 , 95\% CI 1.13 to 6.41 , GRADE: moderate) but did not reach statistical significance regarding downgrading of AIN (RR 15.24, 95\% CI 0.92 to 251.29 , GRADE: low). RRs for intermediate-term and long-term recurrence could not be calculated due to zero events in the five patients whose AIN cleared after EOT. Progressive grade of dysplasia of AIN was seen less often in the imiquimod group, but the difference was not statistically significant (RR $0.31,95 \%$ CI 0.01 to 7.30 , GRADE: moderate). Dropouts due to $\mathrm{AE}$, pain during treatment and local $\mathrm{AE}$ were more common 
Figure 1 Preferred Reporting Items for Systematic Reviews and Meta-Analyses (PRISMA) flowchart depicting the process of study selection. ML, Medline; MLiP, Medline in Process; EB, Embase; CO, Cochrane Library; HPV, human papillomavirus.

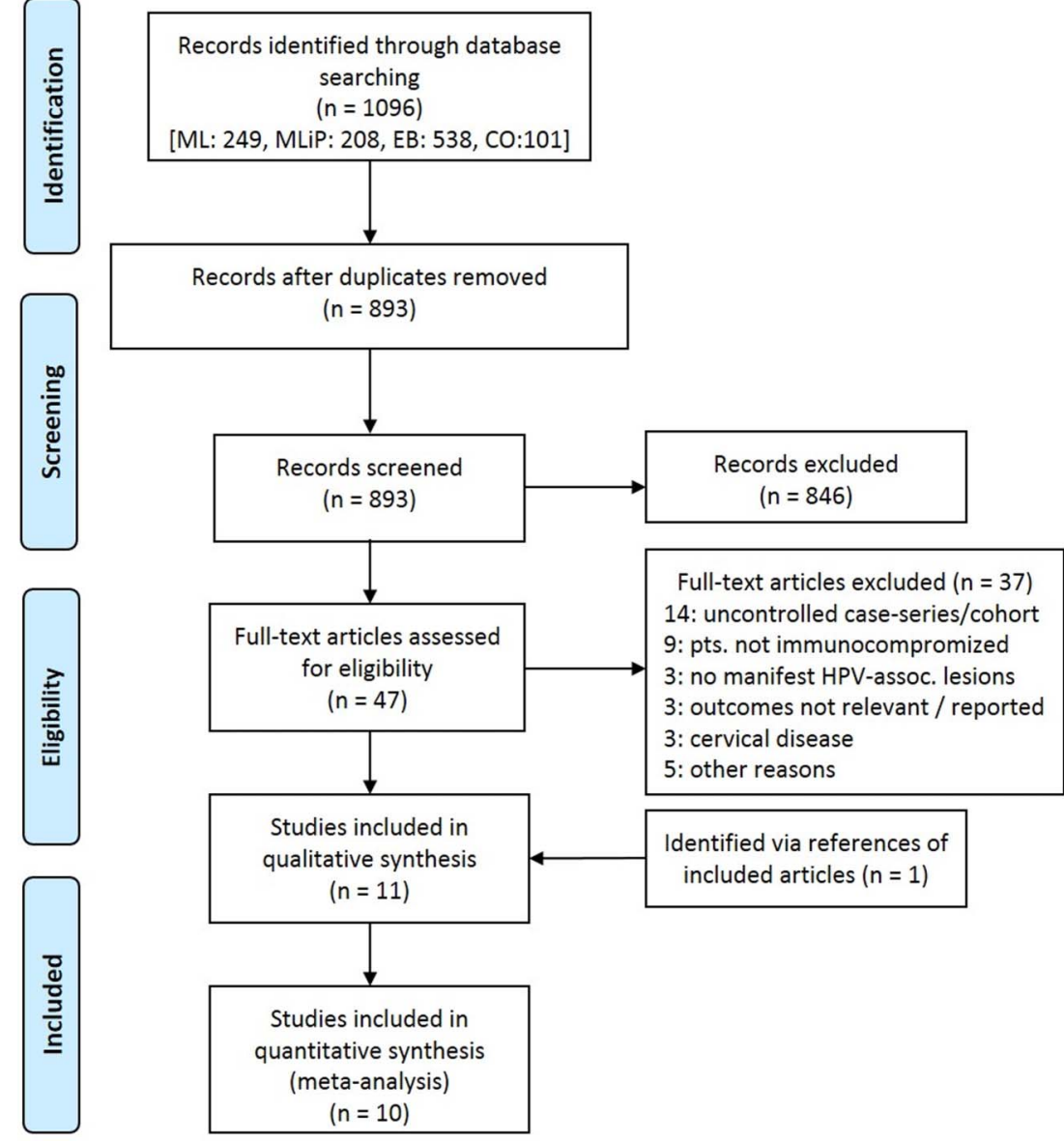

in the imiquimod-treated groups, but not at a statistically significant level (GRADE: low to moderate). Figure 3 shows forest plots for selected efficacy outcomes.

\section{Imiquimod versus electrocautery}

One $\mathrm{RCT}^{8}$ assessed imiquimod 5\% cream and electrocautery in patients with intra-anal and perianal IEN. Histological clearance of intra-anal and perianal IEN was less frequent with imiquimod than with electrocautery, but not at a statistically significant level (RR 0.62, 95\% CI 0.34 to 0.1.12, GRADE: moderate). Separate analysis of intra-anal and perianal IEN demonstrated statistically significant subgroup differences $\left(\chi^{2}=5.41, \mathrm{df}=1, \mathrm{p}=0.02\right)$ : regarding short-term CC, imiquimod was statistically significantly inferior to electrocautery in the treatment of intra-anal IEN (RR 0.47 , 95\% CI 0.24 to 0.92, GRADE: moderate), but not in the treatment of perianal IEN (RR 1.36, 95\% CI 0.75 to 2.45, GRADE: moderate). Histological downgrading occurred less frequently (not statistically significant) with imiquimod than with electrocautery (RR 0.67 , 95\% CI 0.39 to 1.14 , GRADE: moderate). With respect to intermediate-term and long-term recurrence, no statistically significant differences were seen. Dropouts due to $\mathrm{AE}$ and pain during treatment did not differ between the treatment groups (GRADE: moderate). Figure 4 shows forest plots for selected efficacy outcomes.

\section{Imiquimod vs 5-fluorouracil}

The same trial $^{8}$ investigated a third group applying 5fluorouracil $2 \%$ cream, allowing for a direct comparison to imiquimod in participants with intra-anal or perianal IEN.
Although rates of short-term CC, downgrading of high-grade IEN, dropouts due to $\mathrm{AE}$ and pain were higher with imiquimod, no statistically significant differences between the treatment groups were seen for any of the evaluated outcomes (GRADE: moderate). Intermediate-term and long-term recurrence were less frequent with imiquimod, but again not at a statistically significant level (GRADE: moderate).

\section{5-Fluorouracil versus electrocautery}

Comparing 5-fluorouracil 2\% cream with electrocautery, the same trial ${ }^{8}$ demonstrated histological clearance at a statistically significantly lower frequency with fluorouracil than with electrocautery (RR $0.43,95 \%$ CI 0.21 to 0.88 , GRADE: moderate). A separate analysis of intra-anal and perianal IEN did not demonstrate subgroup differences for this comparison $\left(\chi^{2}=1.71, \mathrm{df}=1\right.$, $\mathrm{p}=0.19)$. Histological downgrading occurred less frequently (not statistically significant) with 5-fluorouracil than with electrocautery (RR $0.63,95 \%$ CI 0.37 to 1.06 , GRADE: moderate). With respect to intermediate-term and long-term recurrence, no statistically significant differences were seen, although recurrences occurred at higher rates with fluorouracil. Dropouts due to $\mathrm{AE}$ and pain during treatment did not differ at a statistically significant level between the treatment groups (GRADE: moderate).

\section{Cidofovir versus placebo}

One small $\mathrm{RCT}^{5}$ compared cidofovir 1\% cream with placebo in 12 participants with external AGW. This study was rated as subject to high risk of 'other sources of bias' due to the small 


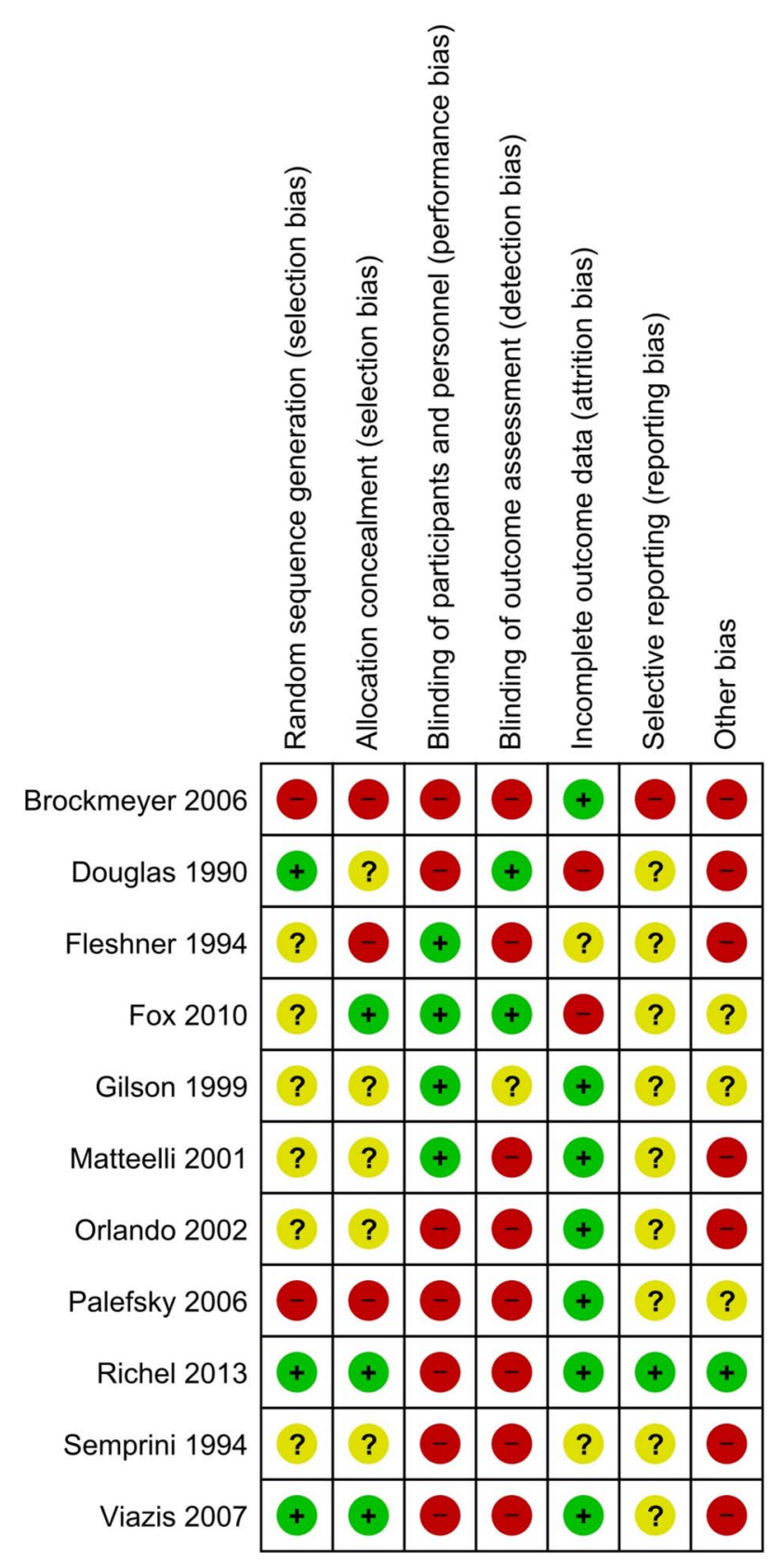

Figure 2 Risk of bias summary. Details on the risk of bias evaluations for each included study.

number of participants and differences in HIV-specific disease parameters, indicating that participants in the placebo group were more severely affected. Although rates of partial clearance, pain during treatment and local $\mathrm{AE}$ were higher in the verum group, no statistically significant differences between the groups were seen (GRADE: very low to low).

\section{Cidofovir versus electrocautery}

An $\mathrm{RCT}^{6}$ compared cidofovir 1\% gel with electrocautery in participants with external AGW. The trial was rated at high risk of 'other sources of bias' due to missing baseline data on disease severity. Short-term CC was less frequent with cidofovir than with electrocautery, but not at a statistically significant level (RR $0.83,95 \%$ CI 0.65 to 1.04 , GRADE: low). However, in those patients whose lesions cleared, intermediate-term recurrence was more common with electrocautery than with cidofovir (RR $0.48,95 \%$ CI 0.24 to 0.96 , GRADE: low).

\section{Systemic IFN- $\alpha$ versus IFN- $\beta$}

One RCT $^{9}$ compared the intramuscular application of IFN- $\alpha$ versus IFN- $\beta$ in women with unspecified 'extensive HPV genital lesions' (p. 136). It was evaluated as being at high risk of 'other sources of bias' because data from only 17 participants who were HIV-positive were included in this review and $>50 \%$ had concurrent cervical dysplasia. No statistically significant difference was seen for intermediate-term CC (GRADE: low). None of the HIV-positive participants dropped out due to AE.

\section{Combined treatment versus single-component treatment}

In a small $\mathrm{RCT}^{10}$ of combined treatment of intra-anal warts with argon plasma coagulation and imiquimod 5\% cream versus argon plasma coagulation alone, no statistically significant differences were seen for short-term CC and long-term recurrence (GRADE: moderate). None of the participants experienced pain or dropped out due to AE. These data were assigned a high risk of 'other sources of bias' due to missing baseline data on disease severity and because data of only a minority of the included patients (HIV-positive: 13/36) were extracted. In the total group (including HIV-negative), the rate of 'mild erythema' at the treatment site was higher in the combined treatment group than with argon plasma coagulation alone (RR 21.84, 95\% CI 1.35 to 353.24, GRADE: moderate).

A three-armed $\mathrm{RCT}^{6}$ compared a combined treatment of cidofovir 1\% gel and electrocautery with cidofovir alone and with electrocautery alone in participants with external AGW. The trial was rated as being at high risk of 'other sources of bias' due to missing baseline data on disease severity. No statistically significant differences of short-term CC (RR 1.06, 95\% CI 0.94 to 1.21 , GRADE: moderate) and intermediate-term recurrence (RR 0.37, 95\% CI 0.14 to 1.01 , GRADE: low) were seen for the combined treatment compared with electrocautery alone. However, statistically significantly more participants experienced short-term CC with the combined treatment compared with cidofovir alone (RR 1.28, 95\% CI 1.03 to 1.61 , GRADE: low). No statistically significant differences were seen for intermediate-term recurrence (RR 0.77 , 95\% CI 0.24 to 2.46, GRADE: low) and local AE (RR 0.75 , 95\% CI 0.34 to 1.66, GRADE: low).

Two RCTs assessed a combination treatment including intralesional application of IFN- $\alpha$ : one of the studies ${ }^{2}$ was rated at high risk of 'other sources of bias' because data of only a minority of participants (HIV-positive: 13/109) were extracted and only three 'test-site warts' (p. 53) were treated. Here, no statistically significant difference was seen between the combination of intralesional IFN- $\alpha-2 b$ with podophyllin $25 \%$ solution and podophyllin alone regarding short-term CC (zero events in both groups, RR not estimable). In the total group (including HIV-negative participants), statistically significantly more participants developed influenza-like symptoms in the group that received IFN (RR 104.50, 95\% CI 6.61 to 1652.40, GRADE: low), data on HIV-positive patients were not reported separately. In the other trial, ${ }^{11}$ also rated as susceptible to 'other sources of bias' because only a minority of participants were HIV-positive (20/43), patients with intra-anal warts either received electrocautery combined with intralesional IFN- $\alpha-n 3$ or electrocautery alone. The rate of recurrences 6 months after initial clearance was lower in the combined treatment group, but this difference was not statistically significant (RR $0.36,95 \%$ CI 0.08 to 1.67 , GRADE: low). 
A

Imiquimod $5 \%$ cream Placebo cream

Risk Ratio

Risk Ratio

1.1.1 External AGW Eyents Total Events

Gilson 1999

Subtotal $(95 \% \mathrm{Cl})$

Total events

Heterogeneity: Not applicable

Test for overall effect: $Z=0.82(P=0.41)$

1.1.2 High grade AIN

Fox 2010 (2)

Subtotal $(95 \% \mathrm{Cl})$

Total events

Heterogeneity: Not applicable

Test for overall effect: $Z=1.17(P=0.24)$

Total $(95 \% \mathrm{Cl})$

Total events

\section{5}

Events

otal Weight M-H, Random, $95 \% \mathrm{C}$

$1.88[0.41,8.59]$
$1.88[0.41,8.59]$

$\mathrm{M}-\mathrm{H}$, Random, $95 \% \mathrm{Cl}$

Heterogeneity: $\mathrm{Tau}^{2}=0.00 ; \mathrm{Chi}^{2}=0.23, \mathrm{df}=1(\mathrm{P}=0.63) ; \mathrm{I}^{2}=0 \%$

Test for overall effect: $Z=1.35(P=0.18$

Test for subgroup differences: $\mathrm{Ch}^{2}=0.23, \mathrm{df}=1(\mathrm{P}=0.63), \mathrm{I}^{2}=0 \%$

$\begin{array}{ll}35 & 66.2 \% \\ 35 & 66.2 \%\end{array}$

(1)

Footnotes

(1) CC after EOT; data solely refer to baseline lesions

(2) CC at 8 weeks after EOT

B

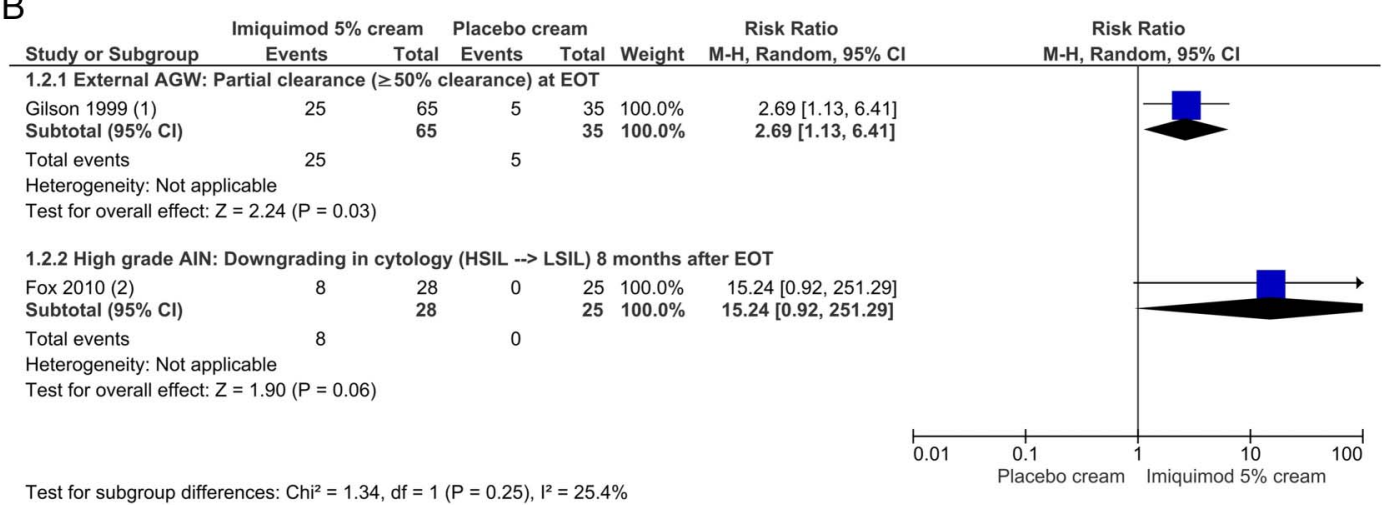

Test for subgroup differences: $\mathrm{Chi}^{2}=1.34, \mathrm{df}=1(\mathrm{P}=0.25), \mathrm{I}^{2}=25.4 \%$

Footnotes

(1) $\geq 50 \%$ clearance; data solely refer to baseline lesions

(2) downgrading in cytology

C

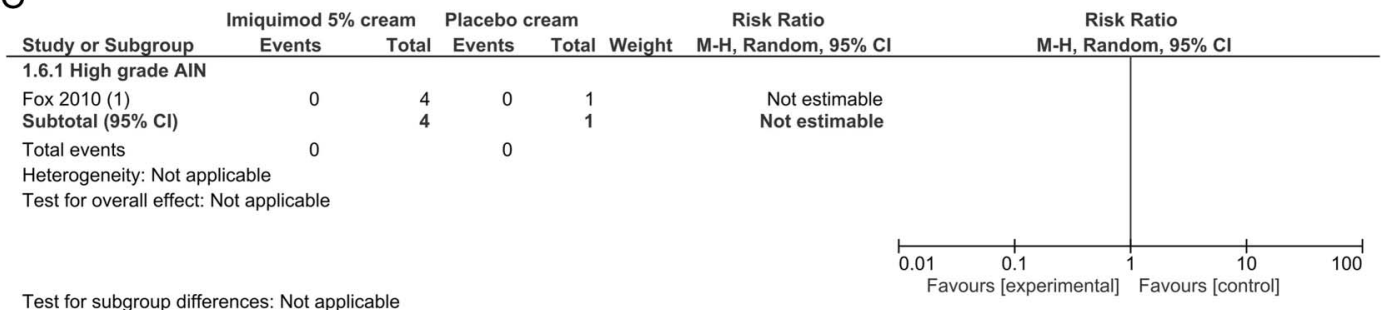

Footnotes

(1) Recurrence at 12 months in pts. who were clear at 8 weeks after EOT

Figure 3 Imiquimod 5\% cream compared with placebo cream: forest plots for selected efficacy outcomes. (A) Short-term complete clearance (CC), (B) reduction in disease severity and (C) long-term recurrence in complete responders. AGW, anogenital warts; AIN, anal intraepithelial neoplasia; EOT, end of treatment; HSIL, high grade squamous intraepithelial lesion; LSIL, low grade squamous intraepithelial lesion; M-H, Mantel-Haenszel.

In a CCT, ${ }^{12}$ two patients were either treated with electrosurgery in combination with systemic IFN- $\alpha-2 b$ or electrosurgery alone. The study was rated as susceptible to 'other sources of bias' because of insufficient information on baseline disease data and because only warts $>5 \mathrm{~mm}$ were electrosurgically treated in both groups. Although more events occurred in the combined treatment group, the differences were not statistically significant concerning short-term CC (RR 7.62, 95\% CI 0.46 to 126.40 , GRADE: very low), $\geq 50 \%$ clearance (RR $14.38,95 \%$ CI 0.93 to 222.06, GRADE: very low), intermediate-term CC (RR 7.62, 95\% CI 0.46 to 126.40 , GRADE: very low) and dropouts due to $\mathrm{AE}$ (RR 4.23, 95\% CI 0.23 to 79.10, GRADE: very low).

\section{Therapeutic vaccination}

One non-randomised study ${ }^{7}$ assessed SGN-00101, a fusion protein of HPV16 E7 protein with Mycobacterium bovis heatshock protein 65 , at three different concentrations in a sample of 15 participants who had high-grade AIN. No placebo control was available. A short-term CC was seen in one of five participants treated with the highest concentration, and in none of the lower concentration groups. Reduction in the grade of dysplasia was seen in two of five participants who received the low and medium concentrations, and in one of five participants who received the high concentration. All of the participants experienced injection-site reactions, but none dropped out due to AE. 


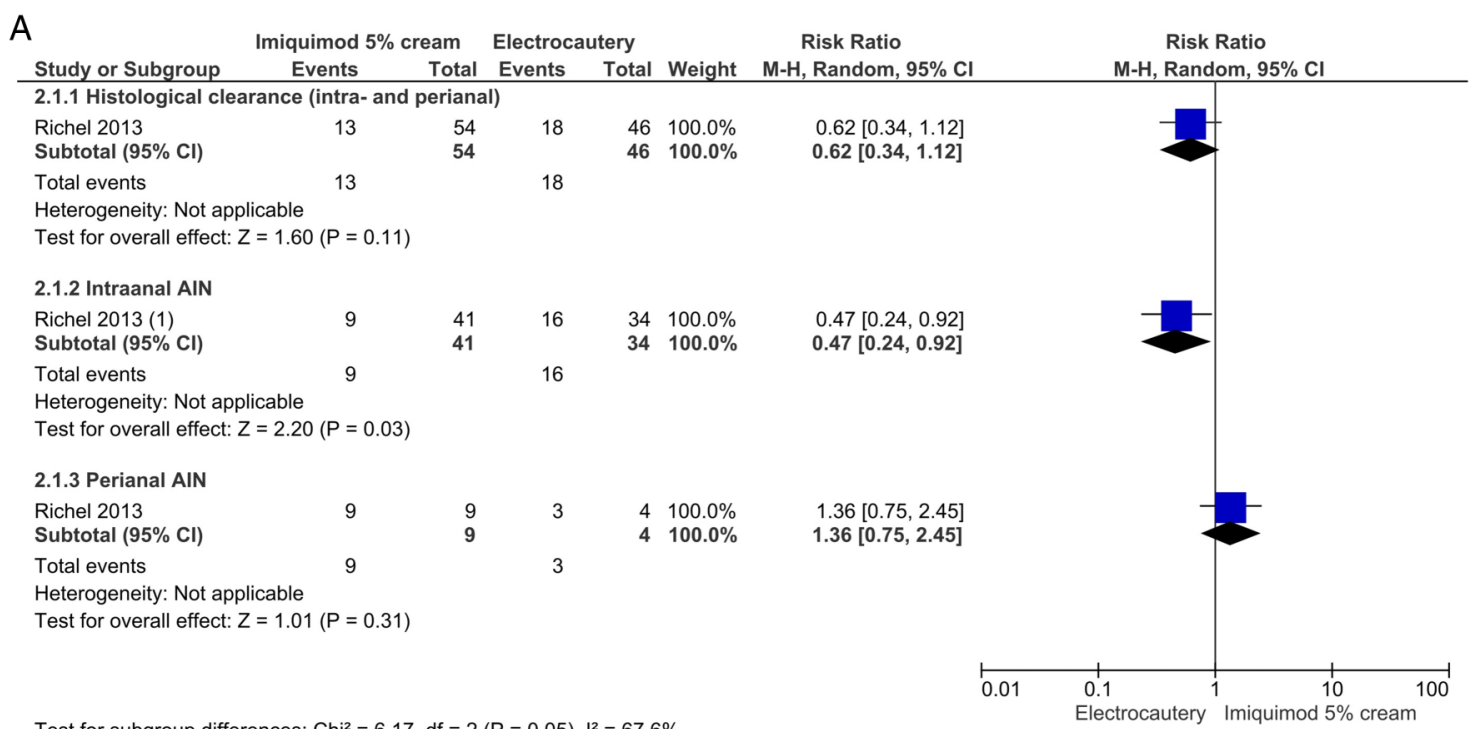

Test for subgroup differences: $\mathrm{Chi}^{2}=6.17, \mathrm{df}=2(\mathrm{P}=0.05), \mathrm{I}^{2}=67.6 \%$ Footnotes

(1) Histological clearance; a non-responder imputation was applied

B

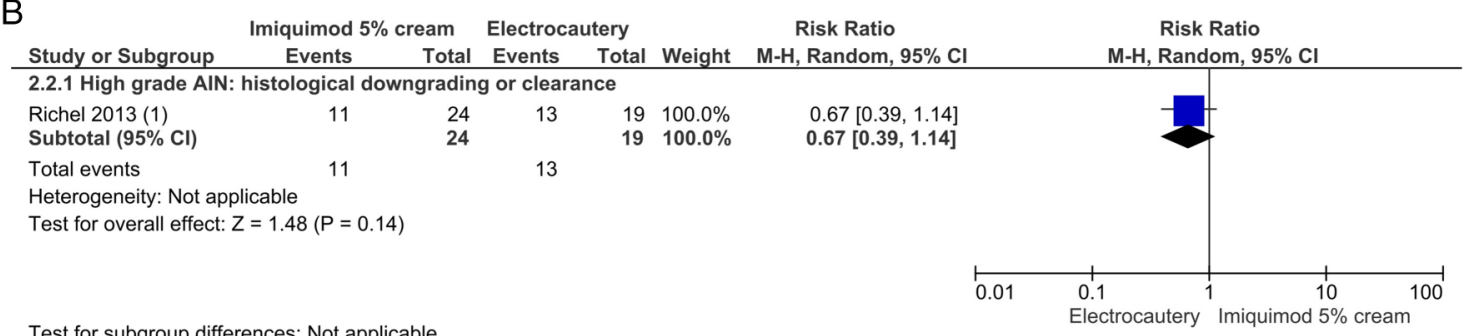

Footnotes

(1) A non-responder imputation was applied

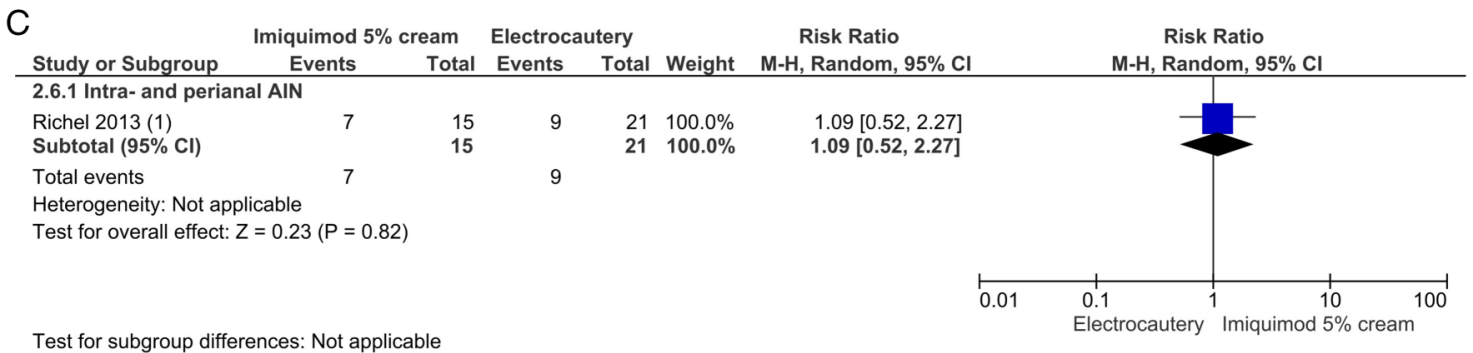

Test for subgroup differences: Not applicable

Footnotes

(1) data include recurrences in those pts. who had clearance or downgrading of lesions

Figure 4 Imiquimod 5\% cream compared with electrocautery: forest plots for selected efficacy outcomes. (A) Short-term complete clearance, (B) reduction in disease severity and (C) long-term recurrence in complete responders. AIN, anal intraepithelial neoplasia.

\section{Additional analyses}

No further analyses such as funnel plots for the detection of publication bias could be performed due to the small number of studies included for each comparison.

\section{DISCUSSION}

Few of the included interventions reached statistically significantly superior efficacy, irrespective of whether compared with placebo or with another active intervention.

For intra-anal IEN, electrocautery proved to be more effective than imiquimod $5 \%$ and 5 -fluorouracil $2 \%$ cream concerning CC 4 weeks after EOT. ${ }^{8}$ This superiority was not seen for perianal IEN and not for downgrading or clearance of high-grade AIN, although downgrading occurred more frequently with electrocautery in both comparisons. Compared with placebo, imiquimod 5\% cream was statistically significantly superior concerning partial clearance in participants who had external warts. ${ }^{4}$ Short-term CC in participants with external warts ${ }^{4}$ and in those who had high-grade $\mathrm{AIN}^{3}$ as well as downgrading in the grade of dysplasia of high-grade AIN were more frequent in the imiquimod than in the placebo groups, but these differences did not reach statistical significance. The combination of electrocautery with cidofovir $1 \%$ gel was superior to cidofovir alone with regard to short-term CC of external AGW; no differences were seen for the other efficacy and safety outcomes. ${ }^{6}$

According to the GRADE evaluations, the overall quality of the evidence ranged from very low to moderate for the comparisons assessed in the included studies. The GRADE quality 
ratings classify our confidence in the appropriateness of the effect estimates, which is important when drawing conclusions from the results. When interpreting the results, in addition to the quality of the evidence, it should be considered that most studies were single-centre studies with a small number of participants in each arm/subgroup. In the absence of sample size calculations, a small number of participants may imply that there was not enough statistical power to detect a difference rather than an overall non-inferiority of the intervention. This should particularly be considered for imiquimod 5\% cream compared with placebo (short-term CC), electrocautery compared with imiquimod $5 \%$ cream and to 5 -fluorouracil $2 \%$ cream (downgrading in grade of dysplasia of AIN), electrocautery compared with cidofovir $1 \%$ gel (short-term CC) and cidofovir $1 \%$ cream or gel compared with placebo (partial clearance).

The studies examined in this review included HIV-positive participants only. We were unable to identify any studies that had included participants with immunodeficiency of other aetiology. While the severity of immunosuppression among HIV-positive patients may greatly vary, the small number of studies included in this review meant that it was not feasible to stratify the analysis by CD4 T-lymphocyte count or HIV disease stage. In the included studies that performed such a stratification, the majority reported no significant association between CD4 count and lesion clearance or reduction. ${ }^{3-68} 811$ Only one study reported a statistically significant association between CD4 counts $>400 / \mathrm{mm}^{3}$ and higher clearance rates when analysing both study groups (intramuscular IFN- $\alpha$ and IFN- $\beta$ ) together $(\mathrm{n}=17)^{9}{ }^{9}$

We did not identify data for some treatment options that have been well-studied and demonstrated efficacy for the treatment of HPV-associated anogenital lesions in immunocompetent patients, such as imiquimod $3.75 \%,{ }^{12}{ }^{13}$ podophyllotoxin $0.5 \%$ solution $^{14-16}$ and sinecatechins $10 \%$ or $15 \%$ ointment. ${ }^{17-19}$ Moreover, we did not identify controlled studies including immunocompromised or HIV-positive patients that investigated established ablational treatment options apart from electrocautery, such as trichloroacetic acid, infrared coagulation or laser treatment.

Data on patient-reported outcomes including QoL were not available in the included studies. This is an important limitation since patient-centred outcomes should play an important role when evaluating treatment strategies. However, apart from the available evidence, the choice of treatment also depends on individual parameters and patient preferences. Patient-reported outcomes should be considered in future research. Drawing conclusions regarding first- and second-line treatment choices for HIV-positive and immunocompromised patients with anogenital HPV-associated lesions is limited. Evidence is scarce, as discussed. In order to recommend specific treatment algorithms, additional factors such as expert opinion, treatment costs and practicability would need to be considered.

\section{CONCLUSIONS}

The available evidence and its interpretation is considerably limited by the often small samples of the included studies. GRADE evaluations of the quality of the evidence ranged from 'very low' to 'moderate'. Evidence was available for the efficacy of electrocautery in the treatment of intra-anal IEN, and imiquimod 5\% cream for the treatment of external AGW. Some further interventions should be subjected to further investigations in larger sample sizes, such as 5-fluorouracil and cidofovir. Interventions established for the treatment in immunocompetent patients such as podophyllotoxin or sinecatechins should also be evaluated in controlled studies for their efficacy in immunocompromised patients. Future research should give attention to these demands and consider patientreported outcomes.

\section{Key messages}

- Immunocompromised and HIV-positive patients carry a high epidemiological burden of anogenital human papillomavirus-associated lesions; it is often difficult to clinically differentiate anogenital warts from intraepithelial neoplasia.

- Evidence for the efficacy of interventions investigated in controlled trials is available for few therapeutic approaches, including electrocautery (anal intraepithelial neoplasia) and imiquimod (external warts).

- The interpretation of statistically insignificant results is limited by small sample sizes and some interventions should be investigated in larger studies.

- Interventions established for treating immunocompetent patients such as podophyllotoxin or sinecatechins should also be evaluated in controlled studies for their efficacy in immunocompromised patients.

\section{Handling editor Jackie A Cassell.}

Contributors RNW, LW, CD and AN developed the research question and methods section. RNW and LW selected eligible studies during the title/abstract-screening and full-text evaluation, extracted data from the included studies, appraised the quality of the evidence, entered and double-checked data for RevMan and analysed/ interpreted the data. AN and CD were involved in cases of dissent with respect to extracted data/evaluations and participated in analysing and interpreting the data and in the final editing of the draft. RNW developed the draft of the manuscript, coordinated contributions of the co-authors and compiled the final draft.

Funding The conduct of the current review was not funded.

Competing interests The Division of Evidence based Medicine (dEBM) received research grants from various entities: The conduct of a previous systematic review and meta-analysis of topical interventions for anogenital warts in immunocompetent patients has been funded with an institutional research grant by Meda Pharma $\mathrm{GmbH} \& \mathrm{Co} . \mathrm{KG}$. The dEBM received research grants not associated with the current work by the Paul-Ehrlich-Gesellschaft für Chemotherapie e.V., European Academy of Dermatology and Venereology, Medigene, European Dermatology Forum, Pfizer, Merz, Deutsche Dermatologische Gesellschaft, Deutsche Gesellschaft für Allergologie und klinische Immunologie, Deutsche Dermatologische Akademie and GSK. The dEBM is currently involved in the development of a German clinical practice guideline on the management of HPV-associated anogenital lesions. AN is responsible for the grants given to dEBM. RNW, LW and CD declare to have no further conflicts of interests. AN has received personal honoraria for educational activity with direct or indirect sponsoring from Novartis, Pfizer, Boehringer Ingelheim, Bayer Healthcare, Jansen and Meda.

Provenance and peer review Not commissioned; externally peer reviewed.

\section{REFERENCES}

1 Brockmeyer NH, Poffhoff A, Bader A, et al. Treatment of condylomata acuminata with pegylated interferon alfa-2b in HIV-infected patients. Eur J Med Res 2006;11:27-32

2 Douglas JM Jr, Eron LJ, Judson FN, et al. A randomized trial of combination therapy with intralesional interferon alpha $2 \mathrm{~b}$ and podophyllin versus podophyllin alone for the therapy of anogenital warts. J Infect Dis 1990;162:52-9.

3 Fox PA, Nathan M, Francis N, et al. A double-blind, randomized controlled trial of the use of imiquimod cream for the treatment of anal canal high-grade anal intraepithelial neoplasia in HIV-positive MSM on HAART, with long-term follow-up data including the use of open-label imiquimod. AIDS 2010;24:2331-5.

4 Gilson RJ, Shupack JL, Friedman-Kien AE, et al. A randomized, controlled, safety study using imiquimod for the topical treatment of anogenital warts in HIV-infected patients. Imiquimod Study Group. AIDS 1999;13:2397-404. 
5 Matteelli A, Beltrame A, Graifemberghi S, et al. Efficacy and tolerability of topical $1 \%$ cidofovir cream for the treatment of external anogenital warts in HIV-infected persons. Sex Transm Dis 2001;28:343-6.

6 Orlando G, Fasolo MM, Beretta R, et al. Combined surgery and cidofovir is an effective treatment for genital warts in HIV-infected patients. AIDS 2002;16:447-50.

7 Palefsky JM, Berry JM, Jay N, et al. A trial of SGN-00101 (HspE7) to treat high-grade anal intraepithelial neoplasia in HIV-positive individuals. AIDS 2006;20:1151-5.

8 Richel O, de Vries HJ, van Noesel CJ, et al. Comparison of imiquimod, topical fluorouracil, and electrocautery for the treatment of anal intraepithelial neoplasia in HIV-positive men who have sex with men: an open-label, randomised controlled trial. Lancet Oncol 2013;14:346-53.

9 Semprini AE, Stillo A, Marcozzi S, et al. Treatment with interferon for genital HPV in HIV-positive and HIV-negative women. Eur J Obstet Gynecol Reprod Biol 1994;53:135-7.

10 Viazis N, Vlachogiannakos J, Vasiliadis K, et al. Earlier eradication of intra-anal warts with argon plasma coagulator combined with imiquimod cream compared with argon plasma coagulator alone: a prospective, randomized trial. Dis Colon Rectum 2007;50:2173-9.

11 Fleshner PR, Freilich MI. Adjuvant interferon for anal condyloma. A prospective, randomized trial. Dis Colon Rectum 1994;37:1255-9.

12 Baker DA, Ferris DG, Martens MG, et al. Imiquimod $3.75 \%$ cream applied daily to treat anogenital warts: combined results from women in two randomized, placebo-controlled studies. Infect Dis Obstet Gynecol 2011;2011:806105.

13 Rosen T, Nelson A, Ault K. Imiquimod cream 2.5\% and 3.75\% applied once daily to treat external genital warts in men. Cutis 2015;96:277-82.

14 Beutner KR, Conant MA, Friedman-Kien AE, et al. Patient-applied podofilox for treatment of genital warts. Lancet 1989;1:831-4.

15 Kirby P, Dunne A, King DH, et al. Double-blind randomized clinical trial of self-administered podofilox solution versus vehicle in the treatment of genital warts. Am J Med 1990;88:465-9.

16 Krogh G, Szpak E, Andersson M, et al. Self-treatment using 0.25\%-0.50\% podophyllotoxin-ethanol solutions against penile condylomata acuminata: a placebo-controlled comparative study. Genitourin Med 1994;70:105-9.

17 Gross $\mathrm{G}$, Meyer KG, Pres $\mathrm{H}$, et al. A randomized, double-blind, four-arm parallel-group, placebo-controlled Phase II/III study to investigate the clinical efficacy of two galenic formulations of Polyphenon $E$ in the treatment of external genital warts. J Eur Acad Dermatol Venereol 2007;21:1404-12.

18 Stockfleth E, Beti H, Orasan R, et al. Topical Polyphenon E in the treatment of external genital and perianal warts: a randomized controlled trial. $\mathrm{Br} J$ Dermatol 2008;158:1329-38.

19 Tatti S, Swinehart JM, Thielert C, et al. Sinecatechins, a defined green tea extract, in the treatment of external anogenital warts: a randomized controlled trial. Obstet Gynecol 2008;111:1371-9. 